\title{
Disorders of intestinal amino-acid transport
}

\author{
M. D. MILNE \\ From the Westminster Medicul School, London
}

There are obvious analogies between disorders of intestinal amino-acid absorption and the more familiar anomalies of carbohydrate absorption. Both monosaccharides and free amino acids are important water-soluble nutrients which must be transported across the lipid-containing intestinal cell membrane by specific active transport processes involving concentration of the compounds within the cell cytoplasm above that of the intestinal lumen or the portal capillary blood. Reduced efficiency of carbohydrate absorption may be due to four possible types of defect: (1) reduced intraluminal hydrolysis of polysaccharides, eg, in pancreatic insufficiency; (2) inefficiency of the active transport process from the lumen to the brush border of the mucosal cell, eg, in hereditary glucose-galactose malabsorption; (3) deficiency or absence of lactase or of sucrase at the brush border of the enterocyte, eg, acquired lactase deficiency; and (4) toxic factors reducing the efficiency of carbohydrate transport, eg, fructose intolerance.

Corresponding factors account for diseases resulting in a reduced efficiency of absorption of the products of protein digestion. One obvious difference between the two types of disease, however, is the more severe diarrhoea produced by carbohydrate malabsorption. Unabsorbed sugars in the colon cause severe osmotic diarrhoea associated with frothy and highly acidic stools. The carbohydrates are partly converted to organic acids within the colonic lumen, and the stool $p \mathrm{H}$ may be lower than 4.0. By contrast, unabsorbed amino acids are almost always in too low concentration to produce osmotic diarrhoea, and their degradation products have not been proved to cause any toxic effects.

\section{Abnormalities of Absorption of the Products of Protein Digestion}

Proteins are hydrolysed in the gut lumen by the combined actions of pepsin, trypsin, chymotrypsin, and carboxypeptidase to a complex mixture of free amino acids and oligopeptides. Free amino acids are absorbed as separate transport groups by a process of active transport, the amino acids being concentrated within the mucosal cell above the amount in the lumen or the portal capillary plasma. Competition occurs between members of the same transport system for absorption. Oligopeptides are taken up by an independent process, and hydrolysed either at the brush border or within the cell cytoplasm to the constituent amino acids. These are then delivered into the portal capillary blood.

\section{REDUCED INTRALUMINAL HYDROLYSIS OF PROTEINS}

Deficiency of pepsin is of little importance and does not apparently cause any absorption defect. By contrast, pancreatic disease may cause severe malabsorption of nitrogenous products, but is not discussed here as only intestinal defects are reviewed. There is, however, an intestinal disorder, recently described, which causes a secondary pancreatic deficit and which was, in fact, initially considered. to be due to primary pancreatic disease. Enterokinase is an enzyme probably located in the brush border of the intestinal mucosal cell which activates trypsinogen in the pancreatic juice. Trypsin then secondarily activates chymotrypsinogen and procarboxypeptidase. Three proved cases of enterokinase deficiency have been reported (Tarlow, Hadorn, Arthur, and Lloyd, 1970a), and probably another three patients previously diagnosed to have trypsin-deficiency disease (Morris and Fisher, 1967; Townes, Bryson, and Miller, 1967) were in actual fact examples of the same condition. There is failure to thrive in infancy, with severe diarrhoea. The stools contain large amounts of unabsorbed nitrogenous compounds. Absorption of amino acids is, however, normal if they are given as mixed free amino acids, and the patients improve if treated with oral pancreatic preparations containing preformed trypsin. Aspirated duodenal juice has no tryptic activity, but this is restored after incubation with human enterokinase. Active enterokinase free from contamination with pancreatic enzymes is best obtained as a duodenal aspirate from a severe case of mucoviscidosis with acinar pancreatic atrophy. Entero- 
kinase deficiency is probably due to autosomal recessive heredity as one case was the child of consanguinous parents.

REDUCED EFFICIENCY OF ACTIVE TRANSPORT OF FREE AMINO ACIDS

Free amino acids are absorbed by at least three active transport systems: (1) many neutral amino acids (absorption is defective in Hartnup disease); (2) dibasic amino acids and cystine (absorption is defective in cystinuria); (3) glycine and the amino acids, proline and hydroxyproline (absorption is defective in some examples of a harmless metabolic anomaly, iminoglycinuria).

In addition, the diacidic amino acids, glutamic and aspartic acids, are largely transaminated to alanine during absorption and there is recent evidence that methionine transport is separate to that of other neutral amino acids.

\section{Hartnup disease}

This is a rare autosomal recessive disease, named after the surname of the first affected family to be reported (Baron, Dent, Harris, Hart, and Jepson, 1956). The basic abnormality of the condition is reduced efficiency of transport of neutral amino acids by the proximal renal tubular cells and by the small intestine (Milne, Crawford, Girao, and Loughridge, 1960). This causes a characteristic aminoaciduria, and nutritional defects from reduced absorption of many essential amino acids. Cases of Hartnup disease are significantly reduced in height (Navab and Asatoor, 1970), and, unless treated with supplements of nicotinamide, are liable to suffer from pellagra due to a deficit of tryptophan with secondary reduction of nicotinamide synthesis. The nutritional defects are, however, mild in degree, and obviously there is no gross deficiency of essential amino acids. Adequate nutrition is, in fact, maintained by a second mechanism of intestinal uptake of essential amino acids which is unimpaired in Hartnup disease. The products of protein digestion are absorbed both by active transport of free amino acids from the gut lumen into the intestinal mucosal cells, and by mucosal uptake of oligopeptides. In Hartnup disease it has been shown that histidine absorption from the dipeptide carnosine, tryptophan from glycyltryptophan, phenylalanine from phenylalanylphenylalanine, and tyrosine from glycyltyrosine are all normal despite a gross deficit of absorption of all these free amino acids (Navab and Asatoor, 1970; Asatoor, Cheng, Edwards, Lant, Matthews, Milne, Navab, and Richards, 1970; Tarlow, Seakins, Lloyd, Matthews, Cheng, and Thomas, 1970b). This confirms other evidence that mucosal uptake of oligopeptides provides a quan- titatively important fraction of the total intestinal 0 absorption of the products of intraluminal digestion.

Cystinuria
The basic defect in cystinuria, a related autosomal recessive disorder, is a similar reduced efficiency of $\frac{}{\circ}$ transport of the basic amino acids and cystine by $\frac{\bar{\sigma}}{\bar{D}}$. the proximal tubular cells of the kidney and by the $\overrightarrow{\widetilde{\sigma}}$ intestinal mucosa (Dent and Rose, 1951; Milne, $\varrho$ Asatoor, Edwards, and Loughridge, 1961). The renal $\%$ defect causes the characteristic disability of the $\overrightarrow{0}$ disease, ie, formation of cystine calculi in the renal pelvis, ureters, or bladder because of the relative $\vec{\omega}$ insolubility of cystine in watery solutions, including $\frac{\Omega}{\circ}$ urine. The intestinal defect causes little disability

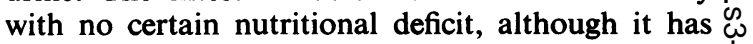
been claimed that the stature of cystinuric patients is slightly reduced as compared to the general popula- $\overrightarrow{-}$ tion (Colliss, Levi, and Milne, 1963), and that the $\vec{\oplus}$

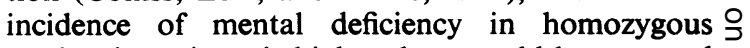
cystinuric patients is higher than would be expected by chance alone (Scriver, Whelan, Clow, and Dallaire, 1970).

In most cystinuric patients, there is no concentration within the mucosal cells of the essential amino acid, lysine, when intestinal biopsy specimens are incubated in Ringer's solution containing addeg . lysine (McCarthy, Borland, Lynch, Owen, an Tyor, 1964; Thier, Segal, Fox, Blair, and Rosenberg 1965). There is thus no active transport mechanism of absorption of this amino acid. Nevertheless, lysine tolerance tests show that increments of plasma lysine are either normal or only slightly depressed (Asatoor, Crouchman, Harrison, Light, Loughridge, Milne, and Richards, 1971). Absorption of the free amino acid can only be by a process of passive or facilitated diffusion not involving any 'uphill' concentrative process from the lumen to the enterocyte. Diffusion mechanisms, therefore, are probably of greater importance in lysine absorption than in the six essential neutral amino acids involved in the defect in Hartnup disease. In addition, mucosal uptake of oligopeptides containing lysine also 을 contributes to the essentially normal transfer of $\rightarrow$ lysine from the gut contents to the portal blood (Hellier, Perrett, and Holdsworth, 1970). Loss of $N$ active transport of free lysine is thus adequately compensated by the presence of at least two alternative mechanisms of lysine uptake by the human gut.

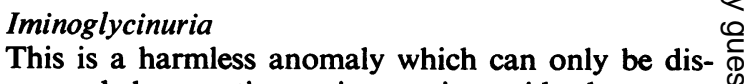
covered by routine urine amino-acid chromato- $\stackrel{?}{+}$ graphy showing the presence of considerable ${ }^{\circ}$ amounts of the two imino acids, proline and hydroxyproline, and of an abnormally high glycine output. 
In most cases of the disorder there has been no demonstrable intestinal defect, but two families have been reported in which there was defective intestinal transport of the three affected amino acids (Morikawa, Tada, Ando, Yoshida, Yokoyama, and Arakawa, 1966; Goodman, McIntyre, and O'Brien, 1967). As none of these compounds are nutritionally essential, it is not surprising that the disorder is symptomless and causes no recognizable disability.

\section{Reduced Intestinal Transport of Single Amino Acids}

In two rare hereditary diseases there is reduced transport of single essential amino acids. In the 'blue diaper syndrome' (Drummond, Michael, Ulstrom, and Good, 1964), a rare cause of infantile hypercalcaemia, there is an isolated defect of tryptophan absorption. Isolated malabsorption of methionine is a rare cause of severe mental deficiency (Hooft, Timmermans, Snoeck, Antener, Oyaert, and van de Hende, 1965). Unabsorbed methionine is degraded by colonic bacteria to $\alpha$-hydroxybutyric acid, which causes a characteristic unpleasant odour in the patients and their excreta. Absorption of methionine from the gut is probably carried out by a separate specific transport system (Bartsocas, Crawford, and Thier, 1970) not involving other amino acids. The parents and some of the sibs of affected cases show a partial defect of methionine transport but have no clinical disability (Hooft, Carton, Snoeck, Timmermans, Antener, van de Hende, and Oyaert, 1968). These isolated defects of amino-acid transport are somewhat paradoxically more serious than are diseases in which groups of amino acids are affected, eg, Hartnup disease and cystinuria. It is, unfortunately, unknown whether the disability is due to a total bodily deficiency of essential amino acids, and in particular whether the absorption of methionine or tryptophan-containing oligopeptides is also impaired.

\section{DEFICIENCY OR ABSENCE OF PEPTIDASES IN} THE ENTEROCYTE

Although peptidases in the intestinal mucosal cells are reduced in gluten-sensitive enteropathy, this is not severe enough to interfere with oligopeptide uptake and hydrolysis. There is, however, some impairment of free amino-acid uptake by intestinal mucosa from patients with coeliac disease and Whipple's disease (Brice, Owen, and Tyor, 1965). Disorders of amino-acid absorption analagous to lactase or sucrase-isomaltase deficiency in carbohydrate absorption either do not exist or have not been recognized to date.
TOXIC FACTORS AND AMINO-ACID

\section{ABSORPTION}

Absorption of many amino acids is significantly reduced in cases of untreated phenylketonuria and maple syrup urine disease (Mackenzie and Woolf, 1959; Linneweh, Ehrlich, Graul, and Hundeshagen, 1963). In the former condition there is a high concentration of phenylalanine in the plasma and other body fluids, whilst in the latter there is a correspondingly high content of the three branched-chain amino acids. Presumably, the involved amino acids diffuse into the intestinal mucosal cells and even into the gut lumen, and competitively inhibit absorption of other amino acids of the same transport group. Absorption improves after the appropriate dietary treatment has reduced the abnormally high plasma content of phenylalanine or of the branched-chain amino acids to normal levels.

In the Fanconi syndrome there is impaired transport of many amino acids from the lumen of the proximal renal tubule into the peritubular capillary blood. A corresponding defect of intestinal function has only been proved to occur in one variety of the condition, ie, in Lowe's syndrome (Lowe, Terrey, and MacLaughlan, 1952; Bartsocas, Levy, Crawford, and Thier, 1969). This is a X-linked recessive hereditary disease causing severe mental deficiency and abnormalities of the anterior chambers of the eyes, and usually terminating in renal failure in late childhood or adolescence. In specific disorders of amino-acid transport, eg, cystinuria and Hartnup disease, there are no known histological abnormalities in either the gut or kidney. By contrast, in Lowe's syndrome there are characteristic changes in both organs. In the intestinal mucosa, goblet cells are degenerate (Saxl, Podhradská, and Tichý, 1970), being only about one quarter of the usual size and in addition their frequency is reduced to only about one fifth the usual number. It is as yet unknown whether the intestinal defect includes a reduced uptake of oligopeptides as well as impaired free amino acid transport.

Pyridoxine deficiency in the experimental animal causes grossly impaired intestinal amino-acid transport (Jacobs and Hillman, 1958; Jacobs, Flaa, and Belk, 1960). In man, a total bodily deficit of pyridoxine is rare, although many conditions occur (Scriver, 1966) in which greater concentrations of pyridoxine than the normal are required to prevent functional defects of the brain and haemopoietic tissue. Malabsorption of amino acids due to pyridoxine deficiency in man has not been described to date.

\section{Conclusions}

Studies of intestinal amino-acid transport in human 
disease have been profitable in extending knowledge, especially in the following respect.

(1) They provide the sole evidence that the mechanism of transport is either identical in the proximal renal tubule and in the small intestine, or at least an important step in transport is the same in the two sites. (2) They provide further evidence that separate groups of amino acids are absorbed by specific transport systems in man as well as in the experimental animal. (3) They give evidence that uptake of oligopeptides by intestinal mucosal cells with subsequent hydrolysis is a quantitatively important mechanism of absorption, and that this is probably a separate process to active transport of free amino acids. (4) They suggest that more than one transport system with differing affinity constants may be involved in the absorption of some individual amino acids.

Nutritional defects from impaired amino-acid absorption have only been proved to be of clinical importance in Hartnup disease, and even here the disability is mild provided the patient is treated with nicotinamide supplements to prevent pellagra. Presumably this is because of the great reserve potential of the human gut. Most of the products of protein digestion are completely absorbed in the first $100 \mathrm{~cm}$ of small intestine, the remaining $5 \mathrm{~m}$ being merely a reserve in the normal subject. Greater lengths of gut may, however, be involved in patients suffering from defects of amino-acid absorption. In addition, there are alternative mechanisms of absorption, ie, separate methods for amino-acid absorption by active transport and mucosal uptake of oligopeptides. Absorption of single amino acids may be mediated by several transport systems, and passive or facilitated diffusion may occur and become quantitatively important when active transport is impaired.

\section{References}

Asatoor, A. M., Cheng, B., Edwards, K. D. G., Lant, A. F., Matthews, D. M., Milne, M. D., Navab, F., and Richards, A. J. (1970). Intestinal absorption of two dipeptides in Hartnup disease. Gut, 11, 380-387.

Asatoor, A. M., Crouchman, M., Harrison, A. R., Light, F. W., Loughridge, L. W., Milne, M. D., and Richards, A. J. (1971). Intestinal absorption of oligopeptides in cystinuria. (In press.)

Baron, D. N., Dent, C. E., Harris, H., Hart, E. W., and Jepson, J. B. (1956). Hereditary pellagra-like skin rash with temporary cerebellar ataxia, constant renal aminoaciduria, and other bizarre biochemical features. Lancet, 2, 421-428.

Bartsocas, C. S., Crawford, J. D., and Thier, S. O. (1970). Demonstration of an independent transport system for L-methionine in rat intestine and kidney. Acta paediat. (Uppsala), 59, 450-451.

Bartsocas, C. S., Levy, H. L., Crawford, J. D., and Thier, S. O. (1969). A deficiency in intestinal amino acid transport in Lowe's syndrome. Amer. J. Dis. Child., 117, 93-95.

Brice, R. S., Owen, E. E., and Tyor, M. P. (1965). Amino acid uptake and fatty acid esterification by intestinal mucosa from patients with Whipple's disease and non-tropical sprue. Gastroenterology, 48, 584-592.

Colliss, J. E., Levi, A. J., and Milne, M. D. (1963). Stature and nutrition in cystinuria and Hartnup disease. Brit. med. J., 1, 590-592.

Dent, C. E., and Rose, G. A. (1951). Amino acid metabolism in cystinuria. Quart. J. Med., ns 20, 205-219.

Drummond, K. N., Michael, A. F., Ulstrom, R. A., and Good, R. A. (1964). The blue diaper syndrome: Familial hypercalcemia and nephrocalcinosis and indicanuria. Amer. J. Med., 37, 928-948.

Goodman, S. I., McIntyre, C. A., and O'Brien, D. (1967). Impaired intestinal transport of proline in a patient with familial iminoaciduria. J. Pediat., 71, 246-249.

Hellier, M. D., Perrett, D., and Holdsworth, C. D. (1970). Dipeptide absorption in cystinuria. Brit. med. J., 4, 782-783.

Hooft, C., Carton, D., Snoeck, J., Timmermans, J., Antener, I., Hende, C. van de, and Oyaert, W. (1968). Further investigations in the methionine malabsorption syndrome. Helv. paediat. Acta, 23, 334-349.

Hooft, C., Timmermans, J., Snoeck, J., Antener, I., Oyaert, W., and Hende, C. van de (1965). Methionine malabsorption syndrome. Ann. paediat. (Bascl), 205, 73-104.

Jacobs, F. A., Flaa, R. C., and Belk, W. F. (1960). Influence of pyridoxine, pyridoxal, pyridoxal phosphate, deoxypyridoxine, and 2,4-dinitrophenol on L-tyrosine transport. Fed. Proc., 19, 183 .

Jacobs, F. A., and Hillman, R. S. L. (1958). Intestinal absorption of the methionine isomers as affected by deoxypyridoxine. $J$. biol. Chem., 232, 445-451.

Linneweh, F., Ehrlich, M., Graul, E. H., and Hundeshagen, H. (1963). Uber den aminosauren-transport bei phenylketonurischer Oligophrenie. Klin. Wschr., 41, 253-255.

Lowe, C. U., Terrey, M., and MacLachlan, E. A. (1952). Organicaciduria, decreased renal ammonia production, hydrophthalmos, and mental retardation; a clinical entity. Amer. J. Dis. Child., 83, 164-184.

McCarthy, C. F., Borland, J. L., Lynch, H. J., Owen, E. E., and Tyor, M. P. (1964). Deficient uptake of basic amino acids and cystine by intestinal mucosa of patients with cystinuria.J. clin. Investo 43, 1518-1524.

Mackenzie, D. Y., and Woolf, L. I. (1959). Maple syrup urine diseas An inborn error of the metabolism of valine, leucine, and iso leucine associated with gross mental deficiency. Brit. med. $J:$, 1, 90-91.

Milne, M. D., Asatoor, A. M., Edwards, K. D. G., and Loughridge, L. W. (1961). The intestinal absorption defect in cystinuria. Gut, 2, 323-337.

Milne, M. D., Crawford, M. A., Girao, C. B., and Loughridge, L. W. (1960). The metabolic disorder in Hartnup disease. Quart. J. Med., ns 29, 407-421.

Morikawa, T., Tada, K., Ando, T., Yoshida, T., Yokoyama, Y., and Arakawa, T. (1966). Prolinuria: deficient intestinal absorption of imino acids and glycine. Tohoku J. exp. Med., 90, 105-116.

Morris, M. D., and Fisher, D. A. (1967). Trypsinogen deficiency disease. Amer. J. Dis. Child., 114, 203-208.

Navab, F., and Asatoor, A. M. (1970). Studies on intestinal absorption of amino acids and a dipeptide in a case of Hartnup disease. Gut, 11, 373-379.

Saxl, O., Podhradská, O., and Tichý, J. S. (1970). Gastro-intestina findings in Lowe's syndrome. Acta paediat. (Uppsala), 59, 453.

Scriver, C. R. (1966). Vitamin B6 dependency syndromes: their larger significance. Pediatrics, 37, 553-555.

Scriver, C. R., Whelan, D. T., Clow, C. L., and Dallaire, L. (1970). Cystinuria: increased prevalence in patients with mental disease. New Engl. J. Med., 283, 783-786.

Tarlow, M. J., Hadorn, B., Arthurton, M. W., and Lloyd, J. K. N (1970a). Intestinal enterokinase deficiency. A newly recognized o disorder of protein digestion. Arch. Dis. Childh., 45, 651-655.

Tarlow, M. J., Seakins, J. W. T., Lloyd, J. K., Matthews, D. M., Cheng, B., and Thomas, A. J. (1970b). Intestinal absorption and biopsy transport of peptides and amino acids in Hartnup disease Clin. Sci., 39, 18P-19P.

Thier, S. O., Segal, S., Fox, M., Blair A., and Rosenberg, L. E. (1965). Cystinuria: Defective intestinal transport of dibasic amino acids and cystine. $J$. clin. Invest., 44, 442-448.

Townes, P. L., Bryson, M. F., and Miller, G. (1967). Further observations on trypsinogen deficiency disease: Report of a second case. J. Pediat., 71, 220-224. 